\title{
Editorial
}

\section{Years of The Journal of Neuroscience}

In January of 1981, the first issue of the first volume of The Journal of Neuroscience was published. To support the new journal of their professional society, past and future presidents of the Society for Neuroscience, including Vernon Mountcastle, Mark Konishi, and Pasko Rakic, contributed articles to the debut issue. Values that were apparent in the first issue, including an embrace of the breadth of neuroscience-from invertebrates to primates, cells to behavior, biochemistry to physiology, and neurons to glia-remain important to the Journal to this day.

To celebrate 4 decades of publication, we have commissioned articles that reflect this history for publication throughout 2021. Many of these articles are from neuroscientists who published their thesis work in the first volume of JNeurosci. In these Progressions articles, they will reflect on how their work and the field has changed across the last 40 years and tell some of the stories behind the studies they published in 1981 .

To kick off a year of celebration with this first issue of 2021, Christophe Leterrier, whose incredible super resolution images of the neuronal cystoskeleton have resulted in new insights into the cell biology of the neuron, has put together a beautiful photoessay and historical primer that shows how important microscopy has been to understanding neurobiology. It is fitting that each of his montages includes a cover image from JNeurosci, some of which were photographed from original issues that were not easy to obtain in the midst of a pandemic. In addition, we have a Progressions article by Vance Lemmon, who published work in the first issue of the first volume of the Journal.

In 1981, I was just beginning my work as a neuroscientist as a high school intern. It is wonderful to see where the field was when I began to read the scientific literature and how much we have learned since then. Please join me in celebrating 40 years of work that was central to creating the discipline of neuroscience.

(D) Marina R. Picciotto

EiC, JNeurosci

https://doi.org/10.1523/JNEUROSCI.2972-20.2020 